\title{
More than a surprise: The bivalency effect in task switching
}

\author{
Paul D. Metzak ${ }^{1,2}$, Beat Meier ${ }^{3}$, Peter Graf ${ }^{4}$, and Todd S. Woodward ${ }^{1,2}$ \\ ${ }^{1}$ Department of Psychiatry, University of British Columbia, Vancouver, Canada \\ ${ }^{2} \mathrm{BC}$ Mental Health and Addiction Research Institute, Vancouver, Canada \\ ${ }^{3}$ Department of Psychology and Center for Cognition, Learning and Memory, University \\ of Bern, Bern, Switzerland \\ ${ }^{4}$ Department of Psychology, University of British Columbia, Vancouver, Canada
}

\begin{abstract}
When switching tasks, if stimuli are presented that contain features that cue two of the tasks in the set (i.e., bivalent stimuli), performance slowing is observed on all tasks. This generalized slowing extends to tasks in the set which have no features in common with the bivalent stimulus and is referred to as the bivalency effect. In previous work, the bivalency effect was invoked by presenting occasionally occurring bivalent stimuli; therefore, the possibility that the generalized slowing is simply due to surprise (as opposed to bivalency) has not yet been discounted. This question was addressed in two task switching experiments where the occasionally occurring stimuli were either bivalent (bivalent version) or merely surprising (surprising version). The results confirmed that the generalized slowing was much greater in the bivalent version of both experiments, demonstrating that the magnitude of this effect is greater than can be accounted for by simple surprise. This set of results confirms that slowing task execution when encountering bivalent stimuli may be fundamental for efficient task switching, as adaptive tuning of response style may serve to prepare the cognitive system for possible future high conflict trials.
\end{abstract}

Keywords: Task switching; Bivalent stimuli; Cognitive control.

When switching tasks, if the presented stimuli are univalent with respect to the current set of tasks (i.e., they carry features specific to only one task in the current set of tasks), under some conditions, these tasks can be performed equally quickly compared to when the same tasks are carried out in a repeated sequence (Allport \& Wylie, 2000; Jersild, 1927; Rogers \& Monsell, 1995; Wylie \& Allport, 2000). However, when stimuli are presented that are bivalent, that is, carry features shared by two tasks in a set of tasks, performance is substantially slowed (Rubin \& Meiran, 2005). For a concrete example, if one task requires naming the colour of letters printed in colour, and the other requires making case decisions about the same letters, coloured letters are bivalent stimuli with respect to the set of tasks, whereas coloured bars or uncoloured letters would be univalent stimuli with respect to the set of tasks.

In previous task switching studies (Grundy et al., 2011; Meier, Rey-Mermet, Woodward, Müri, \& Gutbrod, 2013; Meier, Woodward, ReyMermet, \& Graf, 2009; Rey-Mermet, Koenig, \& Meier, in press; Rey-Mermet \& Meier, 2012a, 2012b; Woodward, Meier, Tipper, \& Graf, 2003; Woodward, Metzak, Meier, \& Holroyd, 2008), we

Correspondence should be addressed to Todd S. Woodward, Room 116, 3rd Floor, 938 W. 28th Ave., Vancouver, British Columbia, Canada V5Z 4H4. E-mail: toddswoodward@gmail.com

The authors would like to thank Lynn Fontanilla, Luka Janicijevic, Alan Lau, Ligaya Allmer, Sarah Flann, Joyce Tse, Patrick Carolan, Alissa Low, and Jessica Ferguson-King for assistance with data collection. 
observed slower responses on univalent stimuli that appeared among bivalent stimuli. Although this slowing may be expected for the univalent stimuli that share features with the bivalent stimuli (Braverman \& Meiran, 2010; Rubin \& Meiran, 2005), we also observed this slowing on univalent stimuli that never shared any features with the bivalent stimuli. We used the term bivalency effect to describe this slowing and postulated that it reflects the adaptive tuning of a response style which is triggered by the presentation of bivalent stimuli (Woodward et al., 2003, 2008). Slowing task execution when encountering bivalent stimuli may be fundamental for efficient task switching, serving to prepare the cognitive system for possible future high conflict trials.

The bivalency effect has been shown to occur irrespective of which tasks are performed (e.g., parity judgements, case judgements, size judgements, colour judgements, and vowel/consonant judgements), what bivalent stimuli are used (e.g., coloured or large/small letters), and whether the stimuli are presented visually or auditorily (Meier et al., 2009). It has also been shown that it does not depend on priming resulting from using the same response keys for both univalent and bivalent stimuli (Rey-Mermet \& Meier, 2012a).

Despite the numerous studies that have investigated the bivalency effect, one critical issue has not yet been directly evaluated: whether or not the bivalency effect is attributable to more than the surprise produced by the appearance of an infrequently occurring and unexpected stimulus. Since the bivalency effect has always been produced by presentation of rarely occurring or unexpected bivalent stimuli, it may have been caused by either the properties of the bivalent stimuli (i.e., contain features that cue two of the tasks in the set), or by the surprise induced by unexpected and/or rare stimuli. The following two experiments were designed to bring resolution to this issue. We expected the full bivalency effect to emerge only under the conditions when the bivalent stimuli cued two of the three to-be-performed tasks, and that this effect would not depend on task or stimulus type.

\section{EXPERIMENT 1}

We hypothesized that the bivalency effect is caused by the occasional presentation of bivalent stimuli, and that the slowing due to the occasional presentation of bivalent stimuli would be greater than the slowing caused by the occasional presentation of unexpected stimuli that are not bivalent with respect to the current set of tasks. Therefore, in Experiment 1 participants were asked to perform two versions of an experiment in which the only difference was whether the occasionally presented stimuli were bivalent or not with respect to the current set of tasks. We also varied the location of the bivalent stimuli within the task triplet (i.e., the three different tasks that were performed in each trial) in order to ensure the bivalency effect was not position specific.

\section{Method}

\section{Participants}

The participants were 147 healthy, English-speaking volunteers ( 86 women and 61 men, mean age $=26.34$ years, $S D=7.95)$. Participants were recruited via advertisements and word of mouth from the community of Vancouver, British Columbia, and participated in exchange for $\$ 10$ per hour. All participants gave informed written consent prior to their participation.

\section{Design}

Participants were assigned randomly to one of three experimental conditions, such that there were 49 participants in each condition. These three experimental conditions differed solely in terms of the position in which bivalent or surprising stimuli would appear (e.g., on the first, second, or third task within the task triplet). In each of these experimental conditions, participants were asked to perform two versions of the experiment. In one version, the participants would encounter stimuli which were surprising and bivalent with respect to the tasks in the set (e.g., the stimuli contained features relevant to two of the to-beperformed tasks), and in the other version, they would encounter stimuli which were unexpected, but did not contain properties that overlapped with any other tasks in the set. We refer to the versions involving stimuli that were both bivalent and surprising as the bivalent version, and the versions involving stimuli that were only surprising (but not bivalent) as the surprising version, corresponding to our hypotheses.

\section{Materials}

The following univalent stimuli were prepared: For the colour decision task, the stimuli were one of 
four shapes — circle, triangle, square, or pentagondisplayed in either blue or red, and matched in pixel size with the letter and numeral stimuli. For the parity decision task, the stimuli were the numerals 2-9, displayed in white, in 60-point Times New Roman font. For the case decision task, the stimuli were the letters a, b, d, e, displayed in white, in either upper or lower case, in 60-point Times New Roman font.

The bivalent colour stimuli were the letters $a, b$, $\mathrm{d}$, e, displayed in either blue or red, in either upper or lower case, in 60-point Times New Roman font. The surprising stimuli were the same four letters $(\mathrm{a}, \mathrm{b}, \mathrm{d}, \mathrm{e})$ displayed in either a checkered or a sliced texture, in either upper or lower case, in 60-point Times New Roman font. For this experiment, all stimuli were displayed on a screen with a black background.

\section{Procedure}

Participants were tested individually and vocal responses were recorded. Vocal responses were employed because previous work (Rey-Mermet \& Meier, 2012a; Woodward et al., 2008) has shown that the response modality does not affect the bivalency effect, and the use of verbal responses avoids response conflict due to the necessity of pressing the same buttons for all tasks. Participants were seated in front of a computer running SuperLab 2.0 (1999, Cedrus Corporation, San Pedro, California) and affixed with a headset voice-key. Voice-key sensitivity was adjusted to the participant's speaking level prior to the start of the experiment. Once the voice-key was adjusted, the experimental instructions appeared on the screen. As in our previous studies, participants were instructed to carry out three different tasks (referred to as a triplet of tasks): making colour judgements (red/blue) about shapes, making parity (odd/even) judgements about digits, and making case (uppercase/lowercase) judgements about letters. Each participant was instructed to respond "red" or "blue" for the colour task, "odd" or "even" for the parity task, and "upper" or "lower" for the case task. Stimuli remained on the screen until the participant made a response, and a blank screen was displayed for $750 \mathrm{~ms}$ following each response. The duration of the intertrial interval, which appeared after each task triplet, was determined by the time taken for the experimenter to type in the vocal responses (approximately $2 \mathrm{~s}$ ). Please see Figure 1 for depictions of three sample triplets from the bivalent and surprising versions of Experiment 1.

Each participant completed one practice block, in order to familiarize themselves with the stimuli and method of responding, and then performed the two versions of the experiment. Version order was counterbalanced between participants. Each experiment consisted of three blocks, wherein each block contained 40 triplets, and each triplet was composed of a colour judgement, a parity judgement, and a case judgement. The task order for each triplet varied depending on which of the three experimental conditions the participant was assigned, and this order was held constant for both versions of the experiment performed by each participant.

In accordance with past work (Meier et al., 2009; Woodward et al., 2003, 2008), for the first and third blocks in each experiment, only univalent stimuli were presented. During the second block of each the experiment, bivalent stimuli (or unexpected stimuli, depending on the experimental version) were presented on eight (i.e., 20\%) of the casedecision trials. These were distributed pseudorandomly among the 40 triplets. The reason the bivalent stimuli were presented rarely instead of consistently on case-decision trials in Block 2 was to allow computation of univalent RTs on the casedecision trials to compare to those from Blocks 1 and 3. The introduction of a baseline condition (Block 1), an experimental condition (Block 2), and a return to baseline condition (Block 3) produced an ABA style experimental design. Participants were not warned that they would be encountering bivalent or surprising stimuli.

\section{Results}

Trials on which the voice-key reacted to extraneous noise or failed to record a response were excluded from further analyses, and also excluded were outliers, which were defined as responses taking longer than $3000 \mathrm{~ms}$ or shorter than $200 \mathrm{~ms}$. Fewer than $1 \%$ of trials were excluded for these reasons. Although the number of incorrect responses was computed and analysed, only data from correct trials were used in the RT analyses. For all analyses of variance (ANOVAs) used to analyse data from Experiment 1, GreenhouseGeisser adjusted degrees of freedom were checked, but any violations of sphericity did not affect the interpretation of results; therefore, the original degrees of freedom are reported. Table 1 
Bivalent

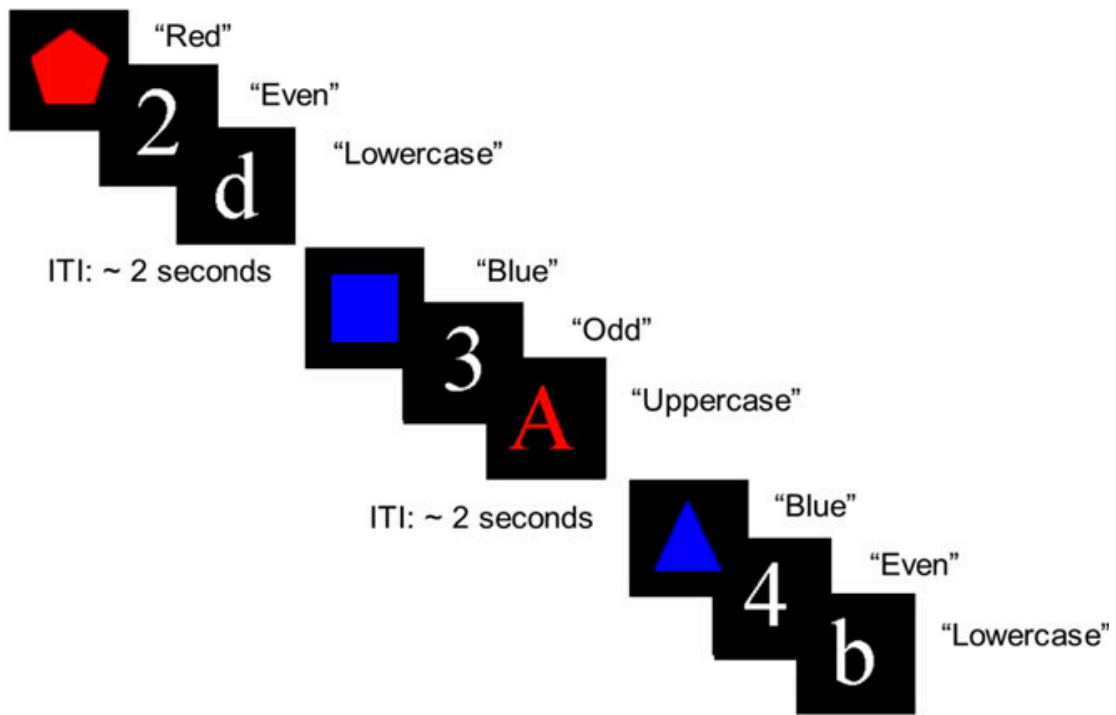

Surprising

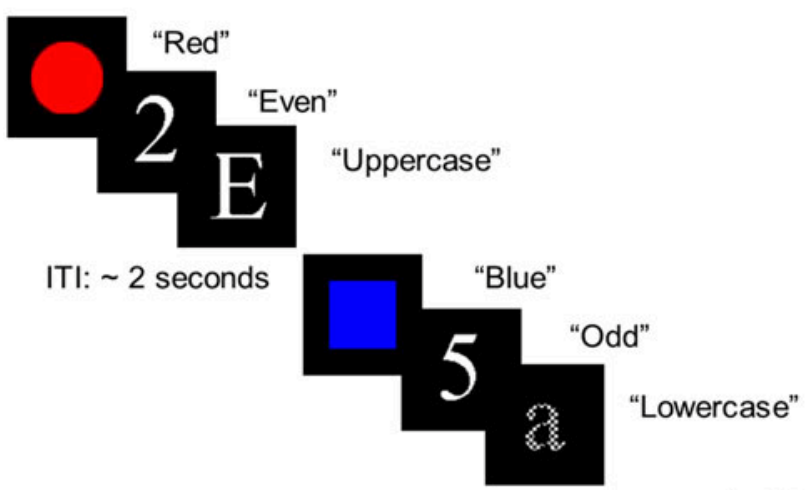

ITI: 2 seconds

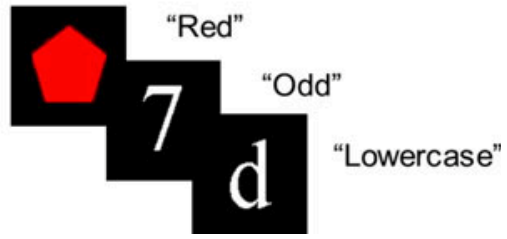

Figure 1. An example of the sequence of events for three consecutive triplets for the bivalent (upper) and the surprising versions (lower) in Experiment 1. Note the "checkered" lowercase "a" in the Surprising example (lower). Texture judgements using "sliced" versus "checkered" shapes replaced colour judgements in the task triplets in Experiment 2, but otherwise the experiment setup was identical to that in Experiment 1.

shows the mean RTs as well as the mean percentage of response errors, separated for the univalent and bivalent trials, and Figure 2 provides a graphical depiction of the main results.

The bivalency effect is defined as the RT slowing in response to univalent stimuli appearing among bivalent stimuli, compared to responses to univalent stimuli in the absence of bivalent stimuli. As in past work (Woodward et al., 2003, 2008), for the analysis of the bivalency effect, polynomial contrasts (linear and quadratic) were used. The quadratic contrast equates to using a block factor with two levels, (1) Block 2 and (2) Blocks 1 and 3 averaged together, and as such, indexes the bivalency effect, so is the primary focus of our analyses. A $2 \times 3 \times 2 \times 3$ repeated measures ANOVA was initially carried out, with the factors of block (Block 2, and the average of Blocks 1 and 3), 
TABLE 1

Mean response times and mean errors presented as a function of experiment version and block in Experiment 1 (standard errors in parentheses); stimuli which are bivalent and surprising occur in the bivalent version, whereas merely surprising stimuli occur in the surprising version

\begin{tabular}{|c|c|c|c|c|c|c|}
\hline \multirow[b]{2}{*}{ Block } & \multicolumn{3}{|c|}{ Bivalent } & \multicolumn{3}{|c|}{ Surprising } \\
\hline & Colour & Parity & Case & Colour & Parity & Case \\
\hline \multicolumn{7}{|c|}{ Response times } \\
\hline \multicolumn{7}{|c|}{ Univalent stimuli } \\
\hline 1 & $646(10)$ & $804(16)$ & $687(12)$ & $644(10)$ & $794(16)$ & $683(12)$ \\
\hline 2 & $684(12)$ & 827 (17) & $706(13)$ & $657(11)$ & $799(16)$ & $689(14)$ \\
\hline 3 & $656(12)$ & 805 (17) & $681(13)$ & $650(11)$ & 798 (16) & $676(14)$ \\
\hline \multicolumn{7}{|c|}{ Bivalent/unexpected stimuli } \\
\hline 2 & & & $796(22)$ & & & 739 (19) \\
\hline \multicolumn{7}{|c|}{ Mean percentage errors } \\
\hline \multicolumn{7}{|c|}{ Univalent stimuli } \\
\hline 1 & $1.09(0.11)$ & $1.89(0.17)$ & $0.96(0.10)$ & $1.23(0.13)$ & $2.04(0.16)$ & $1.17(0.12)$ \\
\hline 2 & $1.05(0.11)$ & $1.67(0.15)$ & $0.69(0.09)$ & $1.08(0.12)$ & $1.78(0.16)$ & $0.84(0.10)$ \\
\hline 3 & $0.90(0.10)$ & $1.53(0.16)$ & $0.88(0.10)$ & $1.01(0.11)$ & $1.54(0.17)$ & $0.88(0.10)$ \\
\hline \multicolumn{7}{|c|}{ Bivalent/unexpected stimuli } \\
\hline 2 & & & $0.72(0.08)$ & & & $0.72(0.05)$ \\
\hline
\end{tabular}

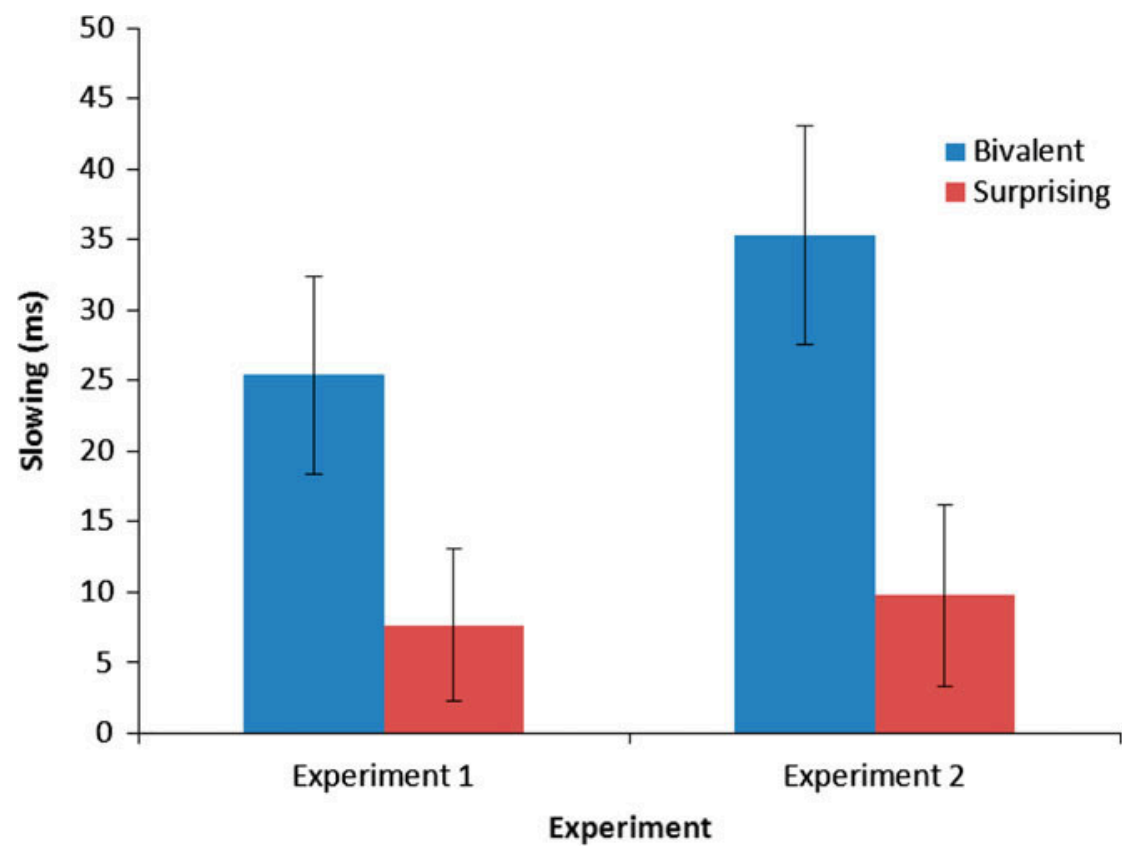

Figure 2. Mean difference in RT slowing between Block 2 and the average of Blocks 1 and 3 plotted as a function of the bivalent and surprising versions of the experiment (standard errors in parentheses). Data from Experiments 1 and 2 are plotted separately.

task (colour, parity, and case judgements), and experiment version (bivalent or surprising) as within-subjects factors, and position (bivalent or surprising stimulus location Position 1,2 , or 3 in the triplet) a between-subjects factor. Only statistical tests involving the Block $\times$ Experiment version interaction are reported here, as this indexes the manipulation of theoretical interest. The four-way interaction was not significant, $F(4,288)=2.23$, $p>.05$, and the three-way interactions involving Block $\times$ Experiment version were also not significant (all $p \mathrm{~s}>.3$ ). In accordance with our expectations, a highly significant Block $\times$ Experiment version interaction emerged, $F(1,144)=$ $15.05, p<.001, \eta^{2}=.10$. This was due to a greater effect of block in the bivalent than the surprising 
version, $F(1,146)=39.49, p<.001, \eta^{2}=.21$, and $F(1,146)=5.89, p<.05, \eta^{2}=.04$, respectively. The block effect produced a $25 \mathrm{~ms}$ RT slowing in the bivalent condition, which replicated the magnitude of the bivalency effect in our previous work. In comparison, the RT slowing attributable to the block effect was $8 \mathrm{~ms}$ in the surprising condition. The error rates were also analysed and no interactions involving Block $\times$ Experiment version reached statistical significance (all $p \mathrm{~s}>.4$ ). The linear contrast, which equates to using a block factor with two levels (Block 1 vs. Block 3), was also analysed by way of a $2 \times 3 \times 2 \times 3$ repeated measures ANOVA. None of the interactions involving the Block $\times$ Experiment version interaction were significant for RTs or errors (all $p \mathrm{~s}>.2$ ).

\section{Discussion}

The results of Experiment 1 demonstrate that the bivalency effect is greater than the effect evoked by simple surprise, and this difference did not interact with task. Additionally, there was no evidence that the bivalency effect was related to the location of the bivalent stimuli within a trial. The results from this experiment suggest bivalent stimuli lead to an RT slowing that is greater than that from stimuli that are merely surprising.

\section{EXPERIMENT 2}

The results of Experiment 1 suggested that the bivalent stimuli induced greater slowing in the subsequent responses to univalent stimuli than did the merely surprising stimuli. However, in order to generalize the findings, the roles of the bivalent and surprising stimuli from Experiment 1 were reversed by replacing the colour judgement task with a texture judgement task in the task triplet in Experiment 2. The goal of this manipulation was to show that the bivalency effect is dependent on stimuli sharing features with two of the tasks in the triplet, and not on the nature of the specific stimuli chosen.

\section{Method}

\section{Participants}

The participants were 144 healthy, English-speaking volunteers ( 89 women and 55 men, mean age = 27.08 years, $S D=8.28$ ). Participants were recruited via advertisements and word of mouth from the community of Vancouver, British Columbia, and participated in exchange for $\$ 10$ per hour. All participants gave informed written consent prior to their participation. None of the participants in Experiment 2 completed Experiment 1.

\section{Design, materials, and procedure}

The design, materials, and procedure for Experiment 2 were identical to those of Experiment 1 with the following exceptions. In Experiment 2, each triplet was composed of a texture judgement, a parity judgement, and a case judgement. For the texture judgement, each participant was instructed to respond "sliced" or "checkered" in response to the texture of the shape that was presented on the screen. The same four shapes used for the colour judgement stimuli in Experiment 1 were used for the texture judgement stimuli in this experiment. In Experiment 2, the bivalent texture stimuli were the letters a, b, d, e, displayed in either a checkered or a sliced texture, in either upper or lower case, in 60-point Times New Roman font, and the surprising stimuli were the same four letters $(\mathrm{a}, \mathrm{b}, \mathrm{d}, \mathrm{e})$ displayed in either a blue or red font, in either upper or lower case, in 60-point Times New Roman font. As in Experiment 1, all participants completed a bivalent and surprising version of the experiment.

As in Experiment 1, participants were assigned to one of three experiments that differed solely on the position of the bivalent (or surprising) stimulus within the triplet. The number of participants in each condition was: (bivalent/surprising stimulus in) first position $=51$, second position $=49$, third position $=44$. Table 2 shows the mean RTs as well as the mean percentage of response errors, separated for the univalent and bivalent trials, and Figure 2 provides a graphical depiction of the main results. Although the number of incorrect responses was computed and analysed, only data from correct trials were used in the RT analyses.

\section{Results}

All data processing and data analyses for Experiment 2 were identical to those performed for Experiment 1, and, as in Experiment 1, fewer than $1 \%$ of trials were excluded due to voice-key errors or extreme response times. Table 2 shows the mean RTs as well as the mean percentage of response errors, separated for the univalent and bivalent trials, and Figure 2 provides a graphical depiction of the main results. 
TABLE 2

Mean response times and mean errors presented as a function of experiment version and block in Experiment 2 (standard errors in parentheses); stimuli which are bivalent and surprising occur in the bivalent version, whereas merely surprising stimuli occur in the surprising version

\begin{tabular}{|c|c|c|c|c|c|c|}
\hline \multirow[b]{2}{*}{ Block } & \multicolumn{3}{|c|}{ Bivalent } & \multicolumn{3}{|c|}{ Surprising } \\
\hline & Texture & Parity & Case & Texture & Parity & Case \\
\hline \multicolumn{7}{|c|}{ Response times } \\
\hline \multicolumn{7}{|c|}{ Univalent stimuli } \\
\hline 1 & $748(12)$ & $826(16)$ & $705(12)$ & $749(12)$ & $815(17)$ & $710(13)$ \\
\hline 2 & $790(14)$ & $867(17)$ & $721(13)$ & $761(12)$ & $827(18)$ & $712(14)$ \\
\hline 3 & $753(13)$ & $818(16)$ & $691(13)$ & $749(12)$ & $815(18)$ & $701(14)$ \\
\hline \multicolumn{7}{|c|}{ Bivalent/unexpected stimuli } \\
\hline 2 & & & $864(26)$ & & & $788(18)$ \\
\hline \multicolumn{7}{|c|}{ Mean percentage errors } \\
\hline \multicolumn{7}{|c|}{ Univalent stimuli } \\
\hline 1 & $1.27(0.13)$ & $1.69(0.14)$ & $1.03(0.11)$ & $1.05(0.12)$ & $1.73(0.15)$ & $0.97(0.11)$ \\
\hline 2 & $1.22(0.12)$ & $1.67(0.14)$ & $0.93(0.12)$ & $1.05(0.11)$ & $1.33(0.10)$ & $0.67(0.07)$ \\
\hline 3 & $0.89(0.10)$ & $1.54(0.14)$ & $0.99(0.12)$ & $0.99(0.11)$ & $1.48(0.14)$ & $0.98(0.10)$ \\
\hline \multicolumn{7}{|c|}{ Bivalent/unexpected stimuli } \\
\hline 2 & & & $0.79(0.11)$ & & & $0.73(0.11)$ \\
\hline
\end{tabular}

Analysing the quadratic contrast (i.e., the bivalency effect), the four-way interaction was not significant, $F(4,288)=1.32, p>.2$, and the threeway interactions involving Block $\times$ Experiment version were also not significant (all $p \mathrm{~s}>.1$ ). In accordance with our expectations, a highly significant Block $\times$ Experiment version interaction emerged, $F(1,141)=24.54, p<.001, \eta^{2}=.15$. This was due to a greater effect size for block in the bivalent relative to the surprising condition, $F(1,143)=65.71, p<.001, \eta^{2}=.32$, and $F(1,143)=$ $7.60, p<.01, \eta^{2}=.05$, respectively. The block effect produced a $36 \mathrm{~ms}$ RT slowing in the bivalent condition, which was comparable to the magnitude of the bivalency effect in our previous work (Woodward et al., 2003, 2008) and in Experiment 1. In comparison, the RT slowing attributable to the block effect was $10 \mathrm{~ms}$ in the surprising condition.

The error rates were also analysed and a significant Block $\times$ Experiment version interaction emerged, $F(1,141)=5.49, p<.05, \eta^{2}=.04$. This was due to a larger effect size for block in the surprising compared to the bivalent version, $F(1$, $143)=5.88, p<.05, \eta^{2}=.04$, and $F(1,143)=0.27$, $p>.60$, respectively. The block effect was characterized by decrease in errors in Block 2 of the surprising version, and a nonsignificant increase in errors in Block 2 of the bivalent version. All other interactions involving the Block $\times$ Experiment version interaction were not significant (all $p \mathrm{~s}>.40$ ). For the linear contrast analysis, which equates to using a block factor with two levels (Block 1 vs. Block 3), no statistical tests involving the Block $\times$ Experiment version interaction were significant for RTs or errors (all $p s>.2$ ).

\section{Discussion}

The results of Experiment 2 replicate those of Experiment 1 in that the slowing characterizing the bivalency effect was found to be greater than the slowing evoked by the introduction of surprising stimuli. The results of Experiment 2 suggest that this effect did not depend on the particular properties of the stimuli themselves, as the same stimuli were used for both Experiments 1 and 2, with only their roles as bivalent or surprising stimuli being reversed.

\section{GENERAL DISCUSSION}

In previous work, we reported that when performing tasks in alternation, generalized slowing occurs when occasional stimuli are presented that contain features that cue two of the tasks in the set (i.e., when stimuli are bivalent as opposed to univalent). This implicated adaptive tuning adjustments to the task set leading to response slowing, and was coined the bivalency effect (Meier et al., 2009; Rey-Mermet \& Meier, 2012a, 2012b; Woodward 
et al., 2003, 2008). Despite the numerous studies that have investigated the bivalency effect, one critical issue has not yet been directly evaluated: whether or not the bivalency effect is attributable to more than the surprise produced by the appearance of an unexpected stimulus. This question was addressed by designing an experiment whereby occasionally presented stimuli were surprising and bivalent in one version of the experiment but only surprising in the other. The results from Experiment 1 and 2 demonstrated that the generalized slowing observed when the occasionally occurring stimuli were bivalent with respect to the task set was much greater than when they were not, and that the bivalency effect occurs regardless of the location of the bivalent stimuli within the task set. This set of experiments suggests that the generalized slowing characteristic of the bivalency effect requires the presentation of stimuli which are bivalent with respect to the current set of tasks, and that this slowing is greater than that observed if merely surprising stimuli are introduced.

The overall pattern of results is consistent between the experiments presented here, as well as our previous research using this paradigm (Meier et al., 2009; Woodward et al., 2003), despite differences in response modality and experimental manipulations. This may result from high-level management of task set execution (Monsell, Yeung, \& Azuma, 2000) in response to the conflict-loaded episodic context of the experiment (Rey-Mermet \& Meier, 2012a) and may be a proactive property of the cognitive system, functional for maintaining accurate performance when processing a currently irrelevant but conflicting stimulus dimension, under the implicit expectation that these "tricky" trials may be encountered in the near future.

One competing hypothesis for the cognitive operations underlying the bivalency effect is that it is due a reactive process whereby the cognitive system adapts to the level of conflict on the previous trial (Gratton, Coles, \& Donchin, 1992; Mayr \& Awh, 2009). This has been addressed as a primary point in two other papers (Meier et al., 2009, 2013). In these papers it was demonstrated that (1) although the bivalency effect declines across trials, it extends well past those immediately following a bivalent stimulus, and (2) a memory-based conflict-loaded context is necessary to produce the bivalency effect. The latter was demonstrated by testing a group of patients with amnesia, for whom a short-lived slowing was present on the task that immediately followed the bivalent stimulus, but with the more enduring slowing that is the essence of the bivalency effect being absent (Meier et al., 2013; cf. Meier \& ReyMermet, 2012, for an elaboration of "episodic context binding"). Thus, although reactivity may increase the magnitude of the bivalency effect in the trial immediately following a bivalent stimulus, the available evidence suggests that the bivalency effect also has a proactive function.

Another competing hypothesis is that the bivalency effect is attributable to the need to inhibit, or undo prior inhibition; however, this is unlikely because slowing was observed on a task that did not share any features with the bivalent stimulus (e.g., the parity judgement task). The task-decision process account (Braverman \& Meiran, 2010; Meiran, 2008) would suggest that the bivalency effect may be related to an increased requirement to refocus attention on the task-salient features of the stimulus; however, since the irrelevant dimension (e.g., the colour/texture of a letter) is never processed on case judgements, refocusing should not be required in this case. The task-set inertia theory (Allport, Styles, \& Hsieh, 1994; Allport \& Wylie, 2000; Koch \& Allport, 2006) would argue that the bivalency effect is based on stimulus-task binding that is exogenously cued by the bivalent stimuli and stimuli that share features with the bivalent stimuli, such that the additional RT reflects the involuntary activation of the irrelevant dimension/task. However, this theory cannot account for the performance slowing found on the task cued by stimuli that do not share features with the bivalent stimulus. Finally, the pattern of results presented here and in our past work (Meier et al., 2009; Woodward et al., 2008) does not support the concept that the bivalency effect can be explained by a speed-accuracy tradeoff, as a decrease in errors has not been reliably associated with the slowing that characterizes the bivalency effect.

In this set of experiments we endeavoured to address one critical issue: whether or not the bivalency effect is attributable to more than the surprise produced by the appearance of an unexpected stimulus. We defined surprising stimuli as infrequently occurring stimuli. Thus, we designed the experiment by controlling for the frequency of occurrence, but manipulating whether or not this 
infrequently occurring stimulus is bivalent with respect to two of the tasks currently in the set of tasks. What constitutes "surprising" stimuli is subject to substantial debate, and a limitation of our study is that our definition is simplified, although it achieves our immediate goals. Further investigation into other possible manipulations of the "surprise" factor could be a topic for future research. The finding that presentation of an infrequent but not bivalent stimulus is insufficient for producing the bivalency effect has been replicated in other work using infrequently presented univalent stimuli (Rey-Mermet \& Meier, in press).

The results of these experiments suggest that (1) the bivalency effect is more than simple surprise, and depends on the presentation of bivalent stimuli, and (2) the bivalency effect occurs regardless of the location of the bivalent stimuli within the task set. The results support an account postulating that the introduction of bivalent stimuli leads to slowing in execution of the entire task set (i.e., the adaptive tuning of response style), due to the presence of at least one form of conflict in the following trials. Slowing task execution when encountering bivalent stimuli may be fundamental for efficient task switching, as adaptive tuning of response style may serve to prepare the cognitive system for possible future high conflict trials.

Original manuscript received May 2012 Revised manuscript received July 2013 Revised manuscript accepted July 2013 First published online September 2013

\section{REFERENCES}

Allport, A., Styles, E., \& Hsieh, S. (1994). Shifting intentional set: Exploring the dynamic control of tasks Attention and performance $X V$ : Conscious and nonconscious information processing (pp. 421-452). Cambridge, MA: MIT Press.

Allport, A., \& Wylie, G. (2000). Task-switching, stimulus-response bindings, and negative priming. In S. Monsell \& J. Driver (Eds.), Attention and performance XVIII:Control of cognitive processes (pp. 35-70). Cambridge, MA: MIT Press.

Braverman, A., \& Meiran, N. (2010). Task conflict effect in task switching. Psychological Research/Psychologische Forschung, 74, 568-578. doi:10.1007/ s00426-010-0279-2

Gratton, G., Coles, M. G. H., \& Donchin, E. (1992). Optimizing the use of information: Strategic control of activation of responses. Journal of Experimental Psychology: General, 121, 480-506. doi:10.1037/00963445.121.4.480
Grundy, J. G., Benarroch, M. F. F., Woodward, T. S., Metzak, P. D., Whitman, J. C., \& Shedden, J. M. (2011). The bivalency effect in task switching: Event-related potentials. Human Brain Mapping, 34, 999-1012. doi:10.1002/hbm.21488

Jersild, A. T. (1927). Mental set and shift. Archives of Psychology, 14(Whole No. 89), (pp. 5-82).

Koch, I., \& Allport, A. (2006). Cue-based preparation and stimulus-based priming of tasks in task switching. Memory and Cognition, 34, 433-444. doi:10.3758/ BF03193420

Mayr, U., \& Awh, E. (2009). The elusive link between conflict and conflict adaptation. Psychological Research, 73, 794-802. doi:10.1007/s00426-008-0191-1

Meier, B., \& Rey-Mermet, A. (2012). Beyond feature binding: Interference from episodic context binding creates the bivalency effect in task switching. [Review]. Frontiers in Psychology, 3. doi:10.3389/fpsyg. 2012.00386

Meier, B., \& Rey-Mermet, A. (in press). An orienting response is not enough: Bivalency not infrequency causes the bivalency effect. Advances in Cognitive Psychology.

Meier, B., Rey-Mermet, A., Woodward, T. S., Müri, R., \& Gutbrod, K. (2013). Episodic context binding in task switching: Evidence from amnesia. Neuropsychologia, 51, 886-892. doi:10.1016/j.neuropsychologia. 2013.01.025

Meier, B., Woodward, T. S., Rey-Mermet, A., \& Graf, P. (2009). The bivalency effect in task switching: General and enduring. Canadian Journal of Experimental Psychology/Revue canadienne de psychologie expérimentale, 63, 201-210. doi:10.1037/a0014311

Meiran, N. (2008). The dual implication of dual affordance: Stimulus-task binding and attentional focus changing during task preparation. Experimental Psychology, 55, 251-259. doi:10.1027/1618-3169.55.4.251

Monsell, S., Yeung, N., \& Azuma, R. (2000). Reconfiguration of task-set: Is it easier to switch to the weaker task? Psychological Research/Psychologische Forschung, 63, 250-264. doi:10.1007/s004269900005

Rey-Mermet, A., Koenig, T., \& Meier, B. (in press). The bivalency effect represents an interference triggered adjustment of cognitive control: An ERP-study. Cognitive, Affective, and Behavioral Neuroscience, 13. doi:10.3758/s13415-013-0160-z

Rey-Mermet, A., \& Meier, B. (2012a). The bivalency effect: Adjustment of cognitive control without response set priming. Psychological Research, 76(1), 50-59. doi:10.1007/s00426-011-0322-y

Rey-Mermet, A., \& Meier, B. (2012b). The bivalency effect: Evidence for flexible adjustment of cognitive control. Journal of Experimental Psychology: Human Perception and Performance, 38, 213-221. doi: 10.1037/a0026024

Rogers, R. D., \& Monsell, S. (1995). Costs of a predictible switch between simple cognitive tasks. Journal of Experimental Psychology: General, 124, 207-231. doi:10.1037/0096-3445.124.2.207

Rubin, O., \& Meiran, N. (2005). On the origins of the task mixing cost in the cuing task-switching paradigm. Journal of Experimental Psychology: Learning, 
Memory, and Cognition, 31, 1477-1491. doi:10.1037/ 0278-7393.31.6.1477

Woodward, T. S., Meier, B., Tipper, C. M., \& Graf, P. (2003). Bivalency is costly: Bivalent stimuli elicit cautious responding. Experimental Psychology, 50, 233-238. doi:10.1026//1618-3169.50.4.233

Woodward, T. S., Metzak, P. D., Meier, B., \& Holroyd, C. B. (2008). Anterior cingulate cortex signals the requirement to break inertia when switching tasks: A study of the bivalency effect. Neuroimage, 40, 13111318. doi:10.1016/j.neuroimage.2007.12.049

Wylie, G., \& Allport, A. (2000). Task switching and the measurement of "switch costs". Psychological Research/Psychologische Forschung, 63, 212-233. doi:10.1007/s004269900003 